\title{
Aerodynamics, thermal management and protection for daily use and race track: Extreme requirements for the new Porsche Panamera
}

Markus Henrich, M. Brzoza, O. Fischer, J. Niewöhner

Dr. Ing. h.c. F. Porsche AG

This manuscript is not available according to publishing restriction. Thank you for your understanding.

() Springer Fachmedien Wiesbaden GmbH 2017

M. Bargende, H.-C. Reuss, J. Wiedemann (Hrsg.), 17. Internationales Stuttgarter Symposium, Proceedings, DOI 10.1007/978-3-658-16988-6_36 